\title{
A Novel Technique for Visualizing Electron Beam Induced Charging
}

\author{
${ }^{1}$ Xiaohu Tang and ${ }^{1,2}$ David C Joy \\ ${ }^{\mathbf{1}}$ Electron Microscope Facility, University of Tennessee, Knoxville, TN 37996-0840 USA \\ ${ }^{2}$ Oak Ridge National Laboratory, Oak Ridge, TN 37831-6064 USA
}

Charging is one of the most important problems encountered in scanning electron microscopy. As a result this phenomena has received a lot of theoretical and experimental attention. Despite this history of work many questions about the nature and behavior of charging remain because of the limitations of the experimental techniques available to study it. For example, although it is now straightforward to determine in situ the surface potential of a sample that is charging during irradiation it is difficult to measure the lateral extent of the charging, or its persistence once the incident beam is switched off. We describe here a simple technique which provides a rapid way of visualizing charging phenomena. ${ }^{[1]}$

The object to be studied (quartz or sapphire) is coated with a 'monolayer' of commercial toner powder of the type used in office copiers. The toner powder is usually applied prior to irradiation, but can also be applied to a specimen recently removed from the microscope. The toner particles are small (micron size), highly electrostatic and lightweight. They readily interact with a field but do not interact with each other. Under the e-beam irradiation, they will be charged and thus sense the presence of the electric field associated with bulk and surface charging and move in the field before coming to rest on the surface at point where the field is a minimum. Once the toner powder has settled it remains firmly attracted to the surface and can be imaged in the SEM.

The surface pre-covered with toner powder is irradiated under $10 \mathrm{keV}$ electron beam on a Hitachi S-3500 SEM under VP mode. Figure 1 shows the sequences of powder patterns which are observed at lower magnification after pre-exposure to the e-beam. This experiment demonstrates how the gas pressure affects the surface electric field. The depleted region both inside and outside the rectangular frame will decrease while the particle cluster will form inside the frame with the increasing gas pressure. Since the toner will be negatively charged by the incident electron beam and the density of the deposit is proportional to electric field intensity, the pattern can indicate how the surface electric field are distributed and what the ir intensity is. Heavier particle density means stronger positive charge while a depleted region corresponds to negative charge. Also the se series of patterns show there is a transition from negative to positive charging between 10 and $15 \mathrm{~Pa}$ gas pressure, which agrees with the charging data determined by measuring the Duane-Hunt (D-H) cut-off of the X-ray spectrum from quartz (Figure 2). Figure 1 also shows that charge field can extent for hundreds of microns away from the charged region under low gas pressure. The pictures (a) and (b) in Figure 1 show a pattern which is suggestive of a flashover caused by dielectric breakdown. Similar images are observed in higher electron beam energy. ${ }^{[2,3]}$ 


\section{References}

[1] The authors are grateful to Prof. B. Griffin (UWA) for valuable suggestions and discussions.

[2] This work was supported by SRC contract LJ-864.003.

[3] Oak Ridge National Laboratory is managed by UT-Batelle, LLC for the USDOE under contract DE-AC05-00OR22725.

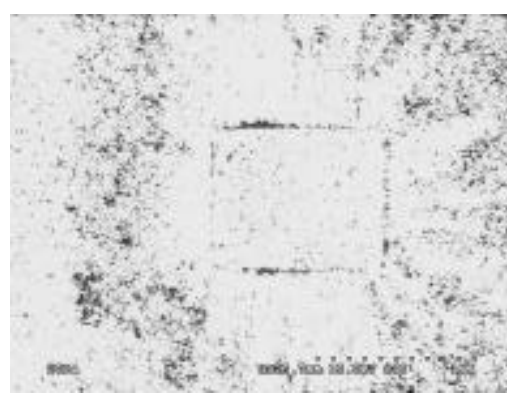

(a) Vacuum

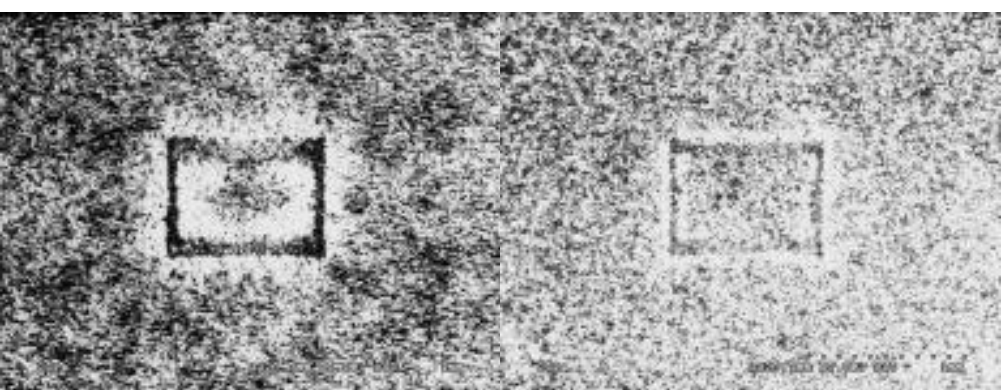

(c) $5 \mathrm{~Pa}$ (e) $10 \mathrm{~Pa}$

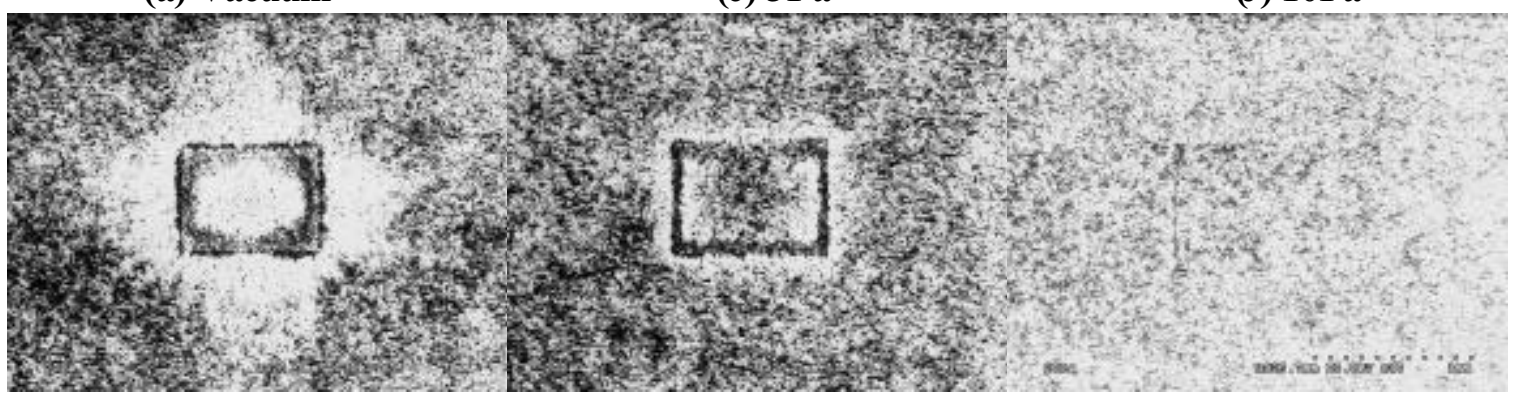

(b) $3 \mathrm{~Pa}$

(d) $7 \mathrm{~Pa}$

(f) $15 \mathrm{~Pa}$

FIG. 1. Toner powder patterns observed on quartz pre-irradiated for 10 seconds by a $10 \mathrm{keV}$ electron beam (magnification 150X) at various ambient gas pressures.

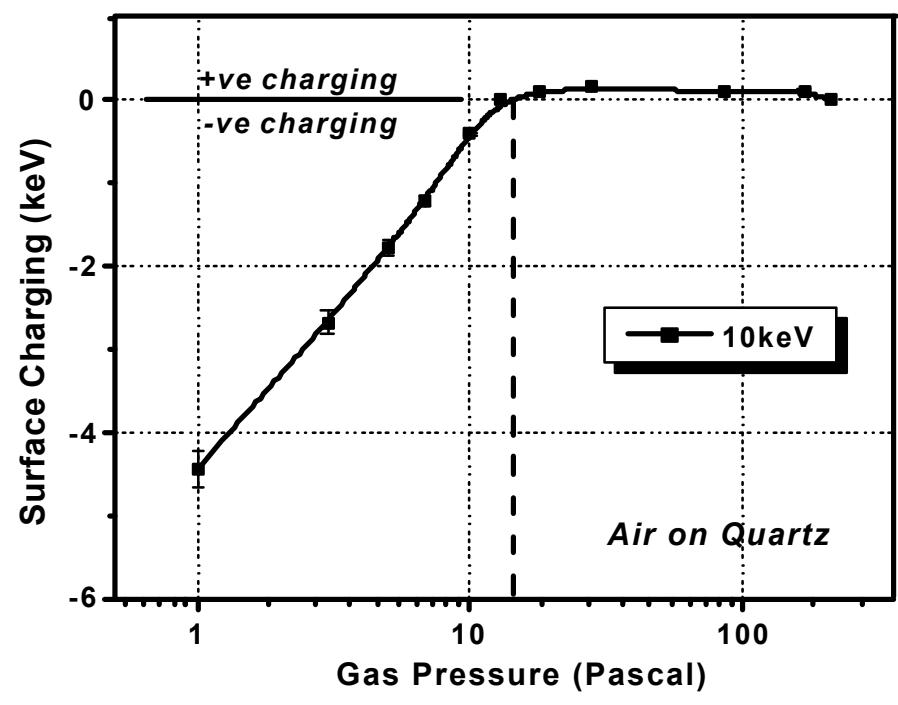

FIG. 2. The measured variation of the surface potential of quartz for the conditions corresponding to the micrographs in figure 1 , note that the condition of gas induced sample neutrality, at $15 \mathrm{~Pa}$ pressure, corresponds to the virtual disappearance of the charge pattern in figure 1 (f). 